\title{
ANÁLISIS ESTADÍSTICO DEL ABSTENCIONISMO EN LAS ELECCIONES PRESIDENCIALES DE COSTA RICA, 2014
}

\author{
Carlomagno Araya Alpízar ${ }^{1}$
}

Recibido: 06/01/2014

Aprobado: 27/02/2015

\begin{abstract}
RESUMEN
En este trabajo se analiza estadísticamente el abstencionismo electoral por distritos $y$ provincias en las elecciones presidenciales de Costa Rica, 2014. El autor estudia la importancia de las variables socioeconómicas como el Índice de Desarrollo Social (IDS), con el objetivo de identificar los factores desencadenantes y que representan un problema de legitimación del sistema político costarricense. En este sentido, se determinó que el abstencionismo electoral en los distritos de Costa Rica se distribuye desigualmente en relación con las características demográficas y económicas; así mismo, tiene una correlación negativa con el índice de desarrollo social y es mayor entre la población con menor edad promedio; además, se presenta con mayor frecuencia en aquellos lugares en donde hay más dependencia económica y baja densidad poblacional, siendo menor en las zonas urbanas. Finalmente, se muestra que el Partido Acción Ciudadana tuvo un efecto positivo en la disminución del abstencionismo.
\end{abstract}

PALABRAS CLAVE: ANÁLISIS ESTADÍSTICO, PARTICIPACIÓN POLÍTICA, ELECCIONES, DIPUTADOS, PRESIDENTE, POLÍTICA, K-MEDIAS.

\section{ABSTRACT}

This paper statistically analyzes the electoral abstention by districts and provinces in the presidential elections of Costa Rica 2014. The author studies the importance of socioeconomic variables as the Social Development Index (IDS), with the aim of studying the factors that originate and represent a problem of legitimacy of the Costa Rican political system. It was found that voter abstention in the districts of Costa Rica is distributed unequally in relation to demographic and economic characteristics, is negatively correlated with the social development index, and is higher among people with lower average age; abstention's frequency was higher in places where there is more economic dependency and is lower in urban areas; districts with low population density tend to have lower electoral participation. In addition, it was found that the Partido Acción Ciudadana had a positive effect in reducing absenteeism.

KEYWORDS: STATISTICAL ANALYSIS, POLITICAL PARTICIPATION, DEPUTIES, PRESIDENT, POLITICS, K-MEANS. 


\section{INTRODUCCIÓN}

El abstencionismo electoral se presenta como uno de los mayores problemas de la democracia costarricense. El fenómeno se ha explicado como consecuencia de las condiciones de pobreza que tienen los electores, además de otras variables como: años de educación, edad, sexo, lugar de residencia y acceso a los servicios públicos. Sin embargo, se debe diferenciar entre dos tipos de abstencionismo: el pasivo y el activo. La abstención pasiva es producto del poco interés en la política, mientras que la abstención activa es una manifestación de rechazo contra la legitimidad de la democracia.

El Diccionario Electoral del Instituto Interamericano de Derechos Humanos (IIDH) menciona que el abstencionismo electoral es "la antitesis o el polo opuesto de la identificación política y de la participación electoral". En este diccionario también se menciona que es "simplemente un signo de conformismo o de un desinterés acomodaticio, de la indolencia y de la ley del mínimo esfuerzo". No hay una única clase de abstención electoral, ni una explicación única. En el primer término, cabe señalar la existencia de una abstención estructural motivada por razones no imputables al ciudadano con derecho a voto: enfermedad, ausencia, defectos de la inscripción censal, clima, alejamiento del colegio electoral, etc. El abstencionismo apático, motivado por la ley del mínimo esfuerzo y el abstencionismo cívico, en el que el ciudadano participa en el acto electoral, pero sin pronunciarse a favor de ninguna de las opciones políticas en pugna, para lo que emite el voto en blanco.

Por lo tanto, el objetivo del trabajo es analizar las variables demográficas y económicas de los distritos y cantones que puedan explicar la variabilidad del abstencionismo. Específicamente, se busca estudiar el nivel de abstención en los distritos de alta y baja participación en las elecciones. Medir la correlación lineal con el índice desarrollo social, tasas de dependencia demográfica y económica, población económicamente activa, etc. La escogencia de las variables se justifica por lo expresado por
Ramírez (2011), "tampoco se ha estudiado la relación entre las características socioeconómicas e históricas de la población en diferentes zonas del territorio nacional y su comportamiento electoral". Además, se elaborará un perfil de los distritos resultantes del análisis de agrupamiento.

El trabajo se desarrolla en las siguientes secciones. En la primera etapa se presenta una revisión de la literatura sobre el abstencionsimo a nivel nacional. A continuación, se presenta los principios básicos del método de construcción de grupos conocido como K-medias y seguidamente se presenta la metodología del estudio. En la cuarta parte, se analizan los resultados usando técnicas estadísticas univariadas y bivariadas. En la última sección se presenta las conclusiones estadísticas del abstencionismo en Costa Rica por distritos en las elecciones presidenciales del 2014.

\section{El abstencionismo electoral}

El director del Centro de Investigación y Estudios Políticos de la Universidad de Costa Rica, Dr. Alberto Cortés, atribuye el abstencionismo a las dificultades logísticas que pueden tener los votantes en estas regiones alejadas de los centros urbanos para llegar a los centros de votación; así como a la decepción que sienten estas comunidades por la política (Córdoba, 2014). En este mismo sentido, Bonilla y Rosero (2006) determinaron que la probabilidad de abstenerse de votar se incrementa en la medida que los lugares de votación se alejan del lugar de residencia. Por otra parte, la abstención está correlacionada al nivel socioeconómico y educativo, de tal forma que los sectores "pobres" se alejan en mayor medida de las urnas. Este tipo de abstencionismo es llamado por Lizama (2012) como pasivo, ya que estos sectores de la sociedad no ejercen su derecho a votar debido a la falta de motivación, en contraste con las personas de clases medias y altas que acuden más a las urnas. En concordancia con lo anterior, Bonilla y Rosero (2006) afirmaron que el abstencionismo depende también de otras varia- 
bles, como el grado de desarrollo de la zona o el nivel educativo.

Hernández (1991) ya había demostrado que Puntarenas y Limón han sido, desde 1953, las provincias de mayor abstencionismo y manifestó su preocupación por el elevado porcentaje, especialmente en Limón. Estas provincias son, a su vez, las regiones de menores niveles de desarrollo y oportunidades para sus pobladores, lo que reafirma la existencia de desigualdades y brechas políticas que se manifiestan regionalmente (Alfaro \& Gómez, 2014).

Por otra parte, en relación con las cualidades psicológicas de los electores que no ejercen el derecho a votar, González (2014) afirma que se caracterizan por ser sujetos pasivos y que no consideran que la política afecte de forma directa su vida, ni que todas las personas tengan la capacidad o posibilidad de participar en política.

Entre otras explicaciones relevantes del abstencionismo, se encuentran la crisis del bipartidismo y el distanciamiento de los ciudadanos de los partidos políticos tradicionales (Vorst, 2009). Además, Raventós, et al. (2005) mencionan que los jóvenes y los hombres se abstienen más frecuentemente que las personas mayores y las mujeres. Finalmente, se afirma que para lograr disminuir los porcentajes de abstencionismo, hay que tomar en consideración variables exploratorias de la variabilidad de la participación electoral; sin embargo, no existe ninguna relación causal lineal entre un único factor y el nivel de participación electoral (Nohlen, 2004).

\section{Método de las K-medias}

El método de las K-medias propuesto por MacQueen en 1967, es un algoritmo para resolver el problema de agrupamiento de individuos o variables en función del grado de similitud medido por la distancia entre ellos. En el caso de este estudio, los individuos corresponderían a los distritos del país, $y$ la variable es el porcentaje de abstencionismo.

El procedimiento que se utiliza para medir la distancia entre distritos, es la distancia euclídea. A saber, la longitud de la recta que une ambos casos y se calcula de la siguiente manera:

$$
d_{i j}{ }^{2}=\sum_{k=1}^{\mathrm{p}}\left(X_{i k}-X_{j k}\right)^{2}
$$

donde $d_{i j}^{2}$ representa la distancia entre los distritos $i$ y $j, X_{i k}$ es el porcentaje de abstención $X_{k}$ para el distrito $i$ y $X_{j k}$ es el porcentaje para el distrito $j$.

La propuesta de Lebart et al. (1995) consiste en establecer puntos iniciales (centroides) que minimicen la inercia intra-grupos, es decir; disminuye la varianza al interior de cada grupo. En cada paso del algoritmo se actualizan los centroides calculados en el paso anterior. El procedimiento consiste en aproximar por etapas un cierto número de grupos (en inglés, cluster) fijados previamente por medio de centroides multivariantes basados en un conjunto de datos de interés. Una vez que todos los casos han sido asignados a uno de los grupos, se inicia un proceso interactivo para calcular los centroides finales de esos conglomerados hasta que ningún caso pueda disminuir la varianza residual.

\section{METODOLOGÍA}

Los resultados de las elecciones se tomaron del Tribunal Supremo de Elecciones de Costa Rica según el cómputo de votos definitivo por distrito, cantón y provincias en las elecciones para Presidente de 2014. La segunda fuente de información es el Ministerio de Planificación Nacional y Política Económica, de donde se obtuvieron los datos sobre la clasificación de los distritos y cantones de Costa Rica según el Índice de Desarrollo Social (IDS) de 2013. Estas fuentes de datos se complementan con las estadísticas demográficas y económicas obtenidas en el Instituto Nacional de Estadística y Censos.

El IDS se usa para clasificar los distritos $y$ cantones del país de acuerdo con su nivel de desarrollo social. Se construye a partir de un conjunto de 11 índices socioeconómicos: Educación (infraestructura educativa, programas 
educativos especiales, escuelas unidocentes y reprobación escolar), participación electoral, salud (bajo peso en niños(as), calidad del agua potable residencial, nacimientos en madres adolescentes solteras), economía (viviendas con acceso a internet, consumo residencial de electricidad) (MIDEPLAN, 2013). Por su parte, los distritos según las categorías de IDS: mayor desarrollo relativo: 70 - 100, nivel medio: 60 - 70 y nivel bajo: menos de 60. En este sentido, es importante recordar que Costa Rica se divide en 7 provincias, 81 cantones y 421 distritos y en la capital (la provincia de San José) es en donde se agrupa el mayor número máximo de cantones y distritos a saber; 20 y 111 respectivamente.

\section{GRÁFICO 1}

DIAGRAMA DE CAJAS PARA EL PORCENTAJE DE ABSTENCIONISMO SEGÚN PROVINCIAS

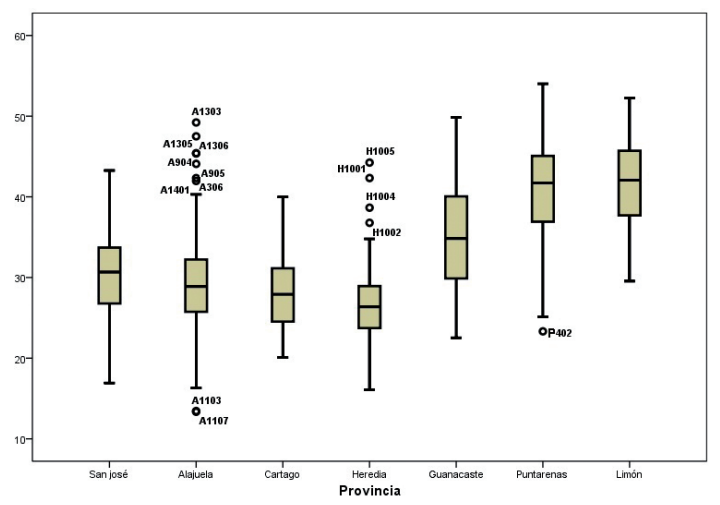

Fuente: Elaboración propia.

\section{ANÁLISIS DE RESULTADOS}

Para analizar los porcentajes de abstencionismo por provincias se presenta el diagrama de cajas gráfico 1. Este describe las estadísticas básicas de los datos e incluye mediana, rango intercuartil (es una medida de variabilidad que se calcula como la diferencia entre el tercer y primer cuartil), identificación de valores extremos (o anormales), naturaleza y magnitud de cualquier desviación de la simetría.

La mediana para el abstencionismo en San José (30.7\%) está muy cercana al porcen- taje observado a nivel nacional; en tanto, en Alajuela (28, 9 \%), Cartago (27, 9 \%) y Heredia $(26,4 \%)$, la mediana decreció por debajo del $30 \%$ de abstención. Por otra parte, en las provincias de Guanacaste (34, $8 \%$ ), Puntarenas (41, 7 \%) y Limón (42, 0 \%), la abstención resultó mayor a la obtenida en la meseta central. En este sentido, Puntarenas y Limón tienen los valores medianos más altos -superiores del $40 \%$-; en otras palabras, en los distritos ubicados en estas dos provincias, la abstención superó el 50\%.

El recorrido intercuartil (altura de la caja) indica la variabilidad de la abstención por distritos y provincias. Se destaca en Cartago la menor dispersión $\left(s_{x}=4,96\right)$ y por lo contrario, la mayor variabilidad se obtiene en Alajuela $\left(s_{x}=6,75\right)$.

En las provincias Alajuela, Heredia y Puntarenas, se presentan porcentaje de abstencionismo anormales o atípicos. Los distritos de Tapesco (A1103) y Las Brisas (A1107) muestran valores muy pequeños dentro de Alajuela. En cambio, San José (A1303), Las Delicias (A1305), Dos Ríos (A1306), Coyolar (A904), La Ceiba (A905), Los Chiles (A1401) y Río Cuarto (A306) alcanzaron niveles de abstención anormalmente altos.

Por otra parte, los distritos de Cureña (H1005), Puerto Viejo (H1001), Llanuras de Gaspar (H1004) y La Virgen (H1002) en Heredia alcanzaron grandes nivel de abstencionismo. En términos generales, en Puntarenas el abstencionismo fue alto; sin embargo, el distrito de La Unión (P402) resultó bastante bajo $(23,32 \%)$.

El porcentaje de abstencionismo por distrito presenta una correlación lineal positiva con la relación de dependencia demográfica $(r=0,62)$, relación de dependencia económica $(r=0,54)$, promedio de hijos e hijas por mujer de 15 años y más $(r=0,61)$ y población masculina $(r=0,58)$. La relación de dependencia demográfica es la relación que existe entre la población dependiente y la población productiva; entonces, esto significa que a mayor dependencia, el porcentaje abstencionismo tiende a crecer en los distritos. 
En este sentido, el incremento de la relación de dependencia económica (relación entre las personas inactivas y las activas) en los distritos produce un aumento en el porcentaje de abstencionismo. Es importante destacar que entre más hombres electores se encuentren en los distritos, el abstencionismo es mayor; es decir, hay una tendencia en estos a no ejercer su derecho a votar. Lo anterior está conforme a lo señalado por Raventós, et al. (2005), quienes concluyen que los hombres tienden a abstenerse en mayor medida que las mujeres.

Por el contrario, el abstencionismo tiene una correlación negativa (inversa) con IDS $(-0,67)$ y edad media de la población $(\mathrm{r}=-0,57)$. Desde la perspectiva del autor de esta investigación, los distritos con menor desarrollo tienden, en mayor proporción, a no acudir a votar, debido a factores como: la lejanía de centro de votaciones, la apatía política, falta de simpatía por los políticos y sus promesas, pues no los ayudan a salir de su situación desventaja económica. Esta tendencia también se observa en jóvenes, quienes se abstienen en mayor medida que las personas mayores. Asimismo, la tasa de ocupación en los distritos tiene una asociación negativa de -0,41 con el abstencionismo y se infiere estadísticamente que cuando aumenta el número de personas con empleos, esta tiende a participar más activamente en las elecciones, lo que provoca la disminución en el porcentaje de abstencionismo.

Es conveniente mencionar que las variables demográficas: porcentaje de población de 0 a 14 años, relación de dependencia demográfica y la relación de dependencia infantil, que corresponden al escenario demográfico de los distritos, tienen una fuerte correlación lineal positiva. Este resultado también se observa en las variables que se refieren al constructo relacionado con la estructura del mercado laboral distrital, representado por la tasa neta de participación, tasa de ocupación, porcentaje de población económicamente inactiva y relación de dependencia económica, las cuales están altamente correlacionas (ver cuadro 1).

CUADRO 1

MATRIZ DE CORRELACIONES LINEALES DE PEARSON ENTRE LAS VARIABLES SOCIOECONÓMICAS

\begin{tabular}{|c|c|c|c|c|c|c|c|}
\hline Variable & $\begin{array}{c}\text { Porcentaje } \\
\text { de } \\
\text { población } \\
\text { de } 0 \text { a } 14 \\
\text { años }\end{array}$ & $\begin{array}{l}\text { Relación de } \\
\text { dependencia } \\
\text { demográfica }\end{array}$ & $\begin{array}{l}\text { Relación de } \\
\text { dependencia } \\
\text { infantil }\end{array}$ & $\begin{array}{l}\text { Tasa neta de } \\
\text { participación }\end{array}$ & $\begin{array}{c}\text { Tasa de } \\
\text { ocupación }\end{array}$ & $\begin{array}{l}\text { Porcentaje } \\
\text { de población } \\
\text { económica } \\
\text { mente inactiva }\end{array}$ & $\begin{array}{c}\text { Relación de } \\
\text { dependencia } \\
\text { económica }\end{array}$ \\
\hline $\begin{array}{l}\text { Porcentaje de } \\
\text { población de } 0 \text { a } \\
14 \text { años }\end{array}$ & 1,000 & & & & & & \\
\hline $\begin{array}{l}\text { Relación de } \\
\text { dependencia } \\
\text { demográfica }\end{array}$ & 0,826 & 1,000 & & & & & \\
\hline $\begin{array}{l}\text { Relación de } \\
\text { dependencia } \\
\text { infantil }\end{array}$ & 0,988 & 0,896 & 1,000 & & & & \\
\hline $\begin{array}{l}\text { Tasa neta de } \\
\text { participación }\end{array}$ & $-0,385$ & $-0,649$ & $-0,451$ & 1,000 & & & \\
\hline $\begin{array}{l}\text { Tasa de } \\
\text { ocupación }\end{array}$ & $-0,390$ & $-0,646$ & $-0,454$ & 0,995 & 1,000 & & \\
\hline
\end{tabular}


Continuación...

\begin{tabular}{|c|c|c|c|c|c|c|c|}
\hline Variable & $\begin{array}{c}\text { Porcentaje } \\
\text { de } \\
\text { población } \\
\text { de } 0 \text { a } 14 \\
\text { años }\end{array}$ & $\begin{array}{l}\text { Relación de } \\
\text { dependencia } \\
\text { demográfica }\end{array}$ & $\begin{array}{l}\text { Relación de } \\
\text { dependencia } \\
\text { infantil }\end{array}$ & $\begin{array}{c}\text { Tasa neta de } \\
\text { participación }\end{array}$ & $\begin{array}{c}\text { Tasa de } \\
\text { ocupación }\end{array}$ & $\begin{array}{l}\text { Porcentaje } \\
\text { de población } \\
\text { económica } \\
\text { mente inactiva }\end{array}$ & $\begin{array}{l}\text { Relación de } \\
\text { dependencia } \\
\text { económica }\end{array}$ \\
\hline $\begin{array}{l}\text { Porcentaje } \\
\text { de población } \\
\text { económicamente } \\
\text { inactiva }\end{array}$ & 0,385 & 0,649 & 0,451 & $-1,000$ & -,0995 & 1,000 & \\
\hline $\begin{array}{l}\text { Relación de } \\
\text { dependencia } \\
\text { económica }\end{array}$ & 0,661 & 0,828 & 0,720 & $-0,928$ & $-0,926$ & 0,928 & 1,000 \\
\hline
\end{tabular}

Fuente: Elaboración propia.

El porcentaje de abstencionismo presenta diferencias estadísticamente significativas según el nivel de desarrollo social. Los distritos con índices más altos de desarrollo $(n=115)$ presentaron una media aritmética de abstención de 25, $6 \%$ y de manera opuesta, aquellos con menor desarrollo social $(n=343)$ tienen una promedio de $34,1 \%$ de abstencionismo, pero alcanzan un valor máximo de 54, $0 \%$. Es importante mencionar que los distritos con bajo desarrollo social representan el 72, $7 \%$ del total de distritos de todo el país.

En el cuadro 2 se aprecia que los mayores niveles de abstencionismo se concentran en los distritos que pertenecen a las provincias de Limón, Puntarenas y Guanacaste, con excepción de los distritos Pizote y Dos Ríos que se ubican en las provincias de San José y Alajuela, respectivamente. Esas provincias concentran los indicadores de la mayor desigualdad económica y social de Costa Rica, especialmente la provincia de Limón. También se desprende de este cuadro que, entre los 10 distritos con los más altos porcentaje de abstencionismo, des- taca Canoas (54, $02 \%)$ perteneciente a Corredores de Puntarenas, y Colorado, ubicado en Pococí de Limón (52, 23 \%). Estos distritos tienen un promedio aritmético de 40,2 en el índice de desarrollo social, valor que los clasifica como de bajo nivel de progreso.

En consonancia con lo planteado por Fernández (2014), en la Sección de Economía y Política del periódico El Financiero, para los expertos la tendencia de altos porcentajes de abstencionismo en los distritos "pobres"; "respalda que una buena parte de la población con menor desarrollo humano es la que se siente más alejada del sistema político y por tanto muestran su indiferencia o decepción ante las ofertas políticas”. En este mismo artículo, el analista político Ricardo Lizano manifiesta que "sigue vigente un descontento con la democracia que no se puede ocultar y que no se ha logrado resolver o disminuir, lo que significa que todavía no hay razones que satisfagan las necesidades o problemas inmediatos de esa población". 
CUADRO 2

COSTA RICA: DISTRITOS CON MAYORES PORCENTAJES DE ABSTENCIONISMO SEGÚN PROVINCIAS E ÍNDICE DE DESARROLLO SOCIAL, ELECCIONES 2014

\begin{tabular}{lccc}
\hline Distrito & Provincia & Índice de Desarrollo Social & Abstencionismo \\
\hline Canoas & Limón & 44,3 & 54,02 \\
Colorado & Limón & 24,8 & 52,23 \\
Golfito & Puntarenas & 49,7 & 51,45 \\
Telire & Limón & 20,7 & 51,18 \\
Santa Cecilia & Guanacaste & 36,0 & 49,87 \\
Sabalito & Puntarenas & 45,7 & 49,68 \\
Pizote & Alajuela & 31,3 & 49,22 \\
La Cuesta & Puntarenas & 53,3 & 48,63 \\
Piedras Blancas & Puntarenas & 34,2 & 48,60 \\
Jacó & Puntarenas & 62,0 & 48,49 \\
\hline
\end{tabular}

Fuente: Elaboración propia.

Contrario al elevado abstencionismo registrado en las provincias periféricas del país, los porcentajes más bajos se registran en las regiones centrales del país: San José, Alajuela, Cartago y Heredia. Sobresalen los distritos de Las Brisas y Tapezco, ambos de la provincia de Alajuela, que alcanzaron solamente un $13 \%$ de abstención (gráfico 2). Entre los 10 distritos con mayor participación política, el índice de desarrollo social en promedio es 76,7, valor considerado por el Ministerio de Planificación Nacional y Política Económica como un alto nivel de desarrollo (MIDEPLAN, 2013).

\section{GRÁFICO 2}

DISTRITOS CON NIVELES MÁS BAJOS DE ABSTENCIONISMO (ALTA PARTICIPACIÓN)

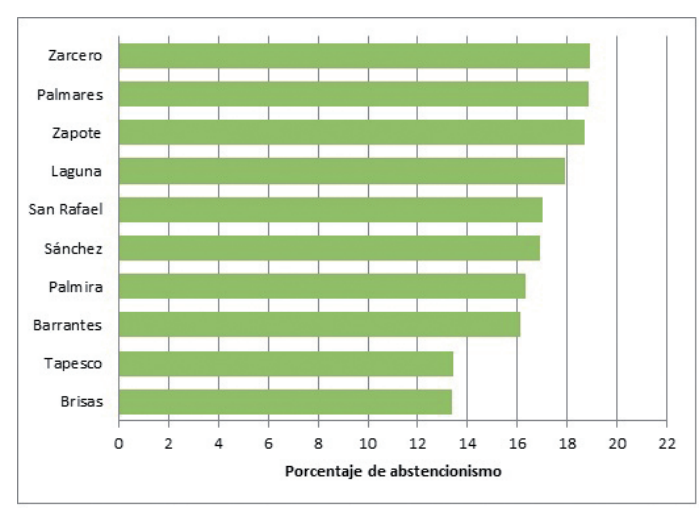

Fuente: Elaboración propia.
Respecto a la correlación entre el porcentaje de abstencionismo y los votos recibidos por los partidos para la elección de presidente, se obtuvo una correlación inversa de $-0,318$ que resulta significativamente diferente de cero, respecto a los votos obtenidos por el Partido Acción Ciudadana (PAC); en tanto que, con los otros partidos no se encontró asociación. Esto implica que al aumentar la votación a favor del PAC, el porcentaje de abstencionismo distrital decreció.

La representación del abstencionismo según el IDS permite identificar la existencia de una geografía del problema político. Los mayores porcentajes de abstención se localizan en las zonas costeras. De esta manera, el abstencionismo está geográficamente asociado a los distritos con bajas condiciones de desarrollo socioeconómicos. Por el contrario, se observa, en el gráfico 2, que en los distritos de la meseta central, en general, se presentan bajas tasas de abstencionismo (en promedio $16,8 \%$ ) y poseen niveles de desarrollo con una media aritmética de 7,6.

Los resultados obtenidos por el método de k-medias, aplicado para determinar el porcentaje de abstencionismo en el nivel de grupos, utilizando como punto de partida tres conglomerados de distritos, nos permite analizar sus características más relevantes. La 
composición de los grupos, obtenidas por este método, se muestra en la cuadro 3 . El primer grupo, al que se ha dado el nombre de bajo abstencionismo, está formado por 187 distritos (39, $5 \%$ ) con un centroide del $25 \%$ de abstención distrital. El grupo 2, caracterizado por un abstencionismo medio, presenta un total de 174 distritos $(36,8 \%)$ y un centroide de 32,7 $\%$, muy semejante al obtenido en la primera ronda del proceso electoral, realizado el 2 de febrero de 2014, en donde el abstencionismo se ubicó en un 31,8 \%. Por último, el grupo 3 está formado por 112 distritos(23,7 \%), se ha catalogado como alto abstencionismo distrital, el mismo tiene un centroide del $42 \%, 8 \%$.

\section{CUADRO 3 \\ CARACTERIZACIÓN DE CONGLOMERADOS DISTRITOS SEGÚN PORCENTAJE DE ABSTENCIONISMO (ANÁLISIS DE K-MEDIAS)}

\begin{tabular}{|c|c|c|c|}
\hline \multirow{2}{*}{ Característica } & \multicolumn{3}{|c|}{ Niveles de Abstencionismo } \\
\hline & Bajo & Medio & Alto \\
\hline Tamaño & 187 & 174 & 112 \\
\hline Porcentaje & $39.5 \%$ & $36.8 \%$ & $23.7 \%$ \\
\hline Centroides & $25,0 \%$ & $32,7 \%$ & $42,8 \%$ \\
\hline
\end{tabular}

Fuente: Elaboración propia.

Los tres grupos de distritos según el IDS $y$ el porcentaje abstencionismo se muestran el gráfico 3. En esta figura, se observa a una nube de puntos que tiene una forma ovoide o elíptica sobre una tendencia lineal de aumentar el abstencionismo, conforme disminuye el IDS en los distritos.

El grupo de bajo abstencionismo (color verde) tiene los mayores niveles en IDS y está representado principalmente, por distritos que pertenecen a las provincias de San José, Alajuela, Cartago y Heredia $^{2}$. El grupo 2, nivel medio de abstencionismo, se caracteriza por un IDS medio de 60 puntos (en una escala de 0 hasta $100)$, cuyos distritos pertenecen a la meseta central del país y algunas jurisdicciones se

$2 \quad$ La primera letra del código del distrito indica la provincia, los siguientes dos dígitos es número de cantón y los últimos son el número de distrito. ubican en Puntarenas y Guanacaste. En tanto, los mayores porcentajes de abstención distrital se ubican en el grupo 3 (alto abstencionismo) $y$ presentan un IDS deficiente, con valores inferiores a los 50 puntos, en su mayoría de la provincia de Limón y Puntarenas.

El agrupamiento de los distritos no corresponde a una distribución geográfica, sino que está determinado el por nivel de desarrollo; por esta razón, se observa en el gráfico 3 que algunos distritos pertenecientes a las cuatros provincias centrales del país tienen altos niveles de abstencionismo, semejantes a las provincias costeras citadas anteriormente.

GRÁFICO 3

REPRESENTACIÓN DE LOS GRUPOS DE DISTRITOS SEGÚN ÍNDICE DE DESARROLLO SOCIAL Y PORCENTAJES DE ABSTENCIONISMO

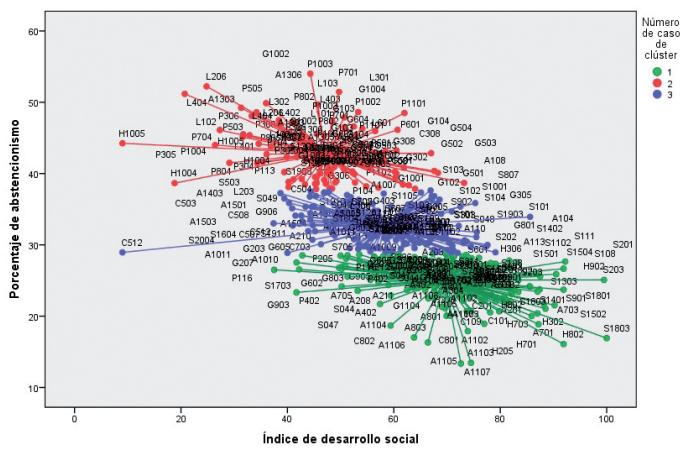

Fuente: Elaboración propia.

Una caracterización más detallada de los grupos de abstencionismo (bajo, medio $y$ alto) determinados por el método de K-Medias, que a su vez considera otras variables socioeconómicas se presenta en el cuadro 4. La media aritmética de votos obtenidos por el Partido Acción Ciudadana (PAC) resulta significativamente diferente por distrito según nivel de abstencionismo. En aquellos distritos en donde este partido obtuvo mayor cantidad media de votos, el nivel de abstencionismo es bajo; por el contrario, el nivel de abstencionismo es alto donde la media aritmética de los votos favorables al PAC es significativamente menor. Se 
desprende de los datos que este partido político tuvo un efecto positivo en el abstencionismo, disminuyéndolo.

Se deduce de los datos analizados que las tasas de abstencionismo están estrechamente asociadas con el IDS de los distritos. Esto significa que los distritos con mayor desarrollo social presentan niveles de abstencionismo bajos, en comparación con aquellos que tienen un menor desarrollo, en donde el nivel de abstención resultó alto. Este es caso de la provincia de Limón, la más rezagada económicamente, olvidada por los Gobiernos de turno y en la cual la población se siente discriminada por el sistema político dominado por población de color blanca de la meseta central. Al respecto, de manera irónica, el expresidente Oscar Arias Sánchez, cuando firma un préstamo con el Banco Mundial (Limón Ciudad Puerto) el 27 de febrero de 2008, afirmaba que: "Limón no será nunca más una provincia olvidada” (Arias, 2008).

La baja densidad poblacional en los distritos dificulta la captación de votos y provoca un incremento en el abstencionismo. En los distritos con altos niveles de abstención, la densidad de la población es de solamente 238, 46 habitantes por $\mathrm{km}^{2}$; en tanto, donde se presentó bajo abstencionismo, la densidad es de 1825, 19 habitantes por $\mathrm{km}^{2}$. Esta diferencia promedio en la densidad de población resulta estadísticamente significativa. Como consecuencia de ello, en las zonas rurales, el problema radica en la dificultad de trasladarse a las mesas de votación. En este sentido, es recomendable disponer de transporte, alimentación y hospedaje gratuito para los ciudadanos de zonas rurales, debido a que los partidos políticos prestan poca atención a estas regiones por el escaso número de personas con derecho a votar.

Asimismo, se observa en el cuadro 4, que en los distritos donde hay más participación electoral, el promedio del porcentaje de población urbana es $65,94 \%$. En tanto, en aquellos distritos donde los niveles de abstención son altos, el promedio resulta en un $34,83 \%$. Estas diferencias promedio en la población urbana por nivel de abstencionismo resultan significativas.
Además, se determinó que hay cuatro indicadores demográficos relacionados con los niveles de abstencionismo, a saber, el porcentaje de población de 0 a 14 años, relación de dependencia demográfica, relación de dependencia infantil $y$ porcentaje de adolescentes madres. Como se observa en el cuadro 4, en los distritos con bajos niveles de abstención, estas variables asumen valores promedio significativamente inferiores a los alcanzados en aquellos distritos con mayores índices de abstencionismo. En el grupo que presenta un alto abstencionismo, hay un 29, $53 \%$ de la población con edades entre los 0 y 14 años, en comparación con 22, $36 \%$ que se ubica en el nivel bajo. Los distritos del grupo "alto abstencionismo" tienen una relación de dependencia demográfica de 55, 29 y una dependencia infantil de 46, 03. También se establece que existen diferencias estadísticamente significativas en los porcentajes promedios de adolescentes madres. En el grupo de distritos que presentaron nivel bajo de abstencionismo, este porcentaje es 2, $50 \%$; en tanto, en zonas de poca participación este valor se duplica $(6,15 \%)$.

Se debe revisar que estos indicadores están estrechamente relacionados a características socioeconómicas de los distritos; en aquellas familias con menores niveles de educación e ingresos, tienden a tener mayores cantidades de hijos, lo cual provoca que los indicadores citados sean mayores a los obtenidos en distritos con bajos porcentaje de abstencionismo.

Para estudiar el comportamiento del abstencionismo, una variable importante a considerar es la edad de los electores. En el cuadro 4 , se muestra que en los niveles altos de abstencionismo distrital, la edad promedio de los electores resulta significativamente inferior a los distritos en donde se presenta mayor participación electoral. Se infiere estadísticamente que el abstencionismo electoral es mayor entre la población de menor edad promedio (los más jóvenes), debido a diversos factores como la apatía por ejercer el derecho a votar y el desinterés por la política.

Además de las variables sociodemográficas relacionadas con el nivel de abstencionismo, existen factores económicos que carac- 
terizan a los distritos con baja participación electoral, los cuales asocian esa actitud de los electores frente a la política tradicional al ausentarse a las mesas de votaciones $y$ permiten intensificar el análisis del problema. Dentro de los factores que inciden en el comportamiento abstencionista están: la tasa neta de participación, la tasa de ocupación, el porcentaje de población económicamente inactiva y la relación de dependencia económica.

\section{CARACTERIZACIÓN DE LOS GRUPOS DE ABSTENCIONISMO CALCULADOS POR EL MÉTODO DE K-MEDIAS SEGÚN ALGUNAS VARIABLES SOCIOECONÓMICAS POR DISTRITO}

\begin{tabular}{|c|c|c|c|c|}
\hline Variable & Grupo & Media aritmética & Error estándar & Sig. \\
\hline \multirow{3}{*}{ Partido Acción Ciudadana } & Bajo & 1894,14 & 145,42 & \multirow{3}{*}{0,000} \\
\hline & Medio & 1282,73 & 146,47 & \\
\hline & Alto & 448,21 & 62,35 & \\
\hline \multirow{3}{*}{ Índice de desarrollo social } & Bajo & 69,58 & 0,86 & \multirow{3}{*}{0,000} \\
\hline & Medio & 58,12 & 0,80 & \\
\hline & Alto & 47,13 & 1,03 & \\
\hline \multirow{3}{*}{ Densidad de población } & Bajo & 1825,19 & 182,88 & \multirow{3}{*}{0,000} \\
\hline & Medio & 1114,46 & 197,88 & \\
\hline & Alto & 238,46 & 96,16 & \\
\hline \multirow{3}{*}{ Porcentaje de población urbana } & Bajo & 65,94 & 2,80 & \multirow{3}{*}{0,000} \\
\hline & Medio & 48,88 & 2,80 & \\
\hline & Alto & 34,83 & 2,72 & \\
\hline \multirow{3}{*}{ Porcentaje de población de 0 a 14 años } & Bajo & 22,36 & 0,24 & \multirow{3}{*}{0,000} \\
\hline & Medio & 25,39 & 0,25 & \\
\hline & Alto & 29,53 & 0,29 & \\
\hline \multirow{3}{*}{ Relación de dependencia demográfica } & Bajo & 44,48 & 0,34 & \multirow{3}{*}{0,000} \\
\hline & Medio & 49,31 & 0,43 & \\
\hline & Alto & 55,29 & 0,61 & \\
\hline \multirow{3}{*}{ Relación de dependencia infantil } & Bajo & 32,41 & 0,40 & \multirow{3}{*}{0,000} \\
\hline & Medio & 38,05 & 0,49 & \\
\hline & Alto & 46,03 & 0,62 & \\
\hline \multirow{3}{*}{ Porcentaje de adolescentes madres } & Bajo & 2,50 & 0,13 & \multirow{3}{*}{0,000} \\
\hline & Medio & 4,22 & 0,18 & \\
\hline & Alto & 6,15 & 0,26 & \\
\hline \multirow{3}{*}{ Tasa neta de participación } & Bajo & 53,71 & 0,41 & \multirow{3}{*}{0,000} \\
\hline & Medio & 50,49 & 0,44 & \\
\hline & Alto & 48,04 & 0,46 & \\
\hline \multirow{3}{*}{ Tasa de ocupación } & Bajo & 52,25 & 0,40 & \multirow{3}{*}{0,000} \\
\hline & Medio & 48,85 & 0,43 & \\
\hline & Alto & 46,43 & 0,44 & \\
\hline
\end{tabular}


Continuación...

\begin{tabular}{lrrrr}
\hline Variable & Grupo & Media aritmética & Error estándar & Sig. \\
\hline \multirow{2}{*}{ Porcentaje de población económicamente } & Bajo & 46,29 & 0,41 & \\
inactiva & Medio & 49,51 & 0,44 & 0,000 \\
& Alto & 51,96 & 0,46 & \\
Relación de dependencia económica & Bajo & 1,44 & 0,02 & \\
& Medio & 1,70 & 0,03 & 0,000 \\
& Alto & 1,99 & 0,04 & \\
\hline
\end{tabular}

Fuente: Elaboración propia.

La tasa neta de participación es el porcentaje de la fuerza de trabajo con respecto a la población de 12 años o más, que para el segundo trimestre del 2013 representaba 2, 23 millones de personas en Costa Rica; en tanto, la población fuera de la fuerza de trabajo se ubicó en 1, 32 millones de personas (INEC, 2013). Entre los grupos de abstencionismo se presentan diferencias promedio significativas; mientras en los distritos baja el nivel abstención, el porcentaje promedio resultó ser 53,71 $\%$ y en los distritos con alto abstencionismo fue de 48, 04. De forma complementaria, la tasa de ocupación que es el porcentaje de la población ocupada (1,99 millones de personas en el segundo semestre del 2013) con respecto a la población de 12 años o más, también presenta igual comportamiento entre los grupos, donde los distritos de mayor participación en las elecciones tienen una tasa promedio de 53, $25 \%$, en comparación al 46, $43 \%$ del grupo de alto abstencionismo.

Esta diferencia en los indicadores entre los niveles de abstencionismo, se refleja en el porcentaje de población económicamente inactiva (cuadro 4), en donde en el grupo de bajo abstencionismo el promedio es 46, $29 \%$; mientras que en los distritos que se ubican en grupo alto abstencionismo, la media aritmética es $51,96 \%$.

Finalmente, otro indicador de los distritos es la relación de dependencia económica, el cual se refiere a la población económicamente inactiva (incluye la población de 0 a 14 años y la de 65 años y más) y la población económicamente activa (ocupados más desempleados). La relación de dependencia econó- mica también muestra significativas promedio entre los diversos grupos de abstencionismo. En los distritos con bajo abstencionismo, la relación de dependencia económica se eleva a 144 personas dependientes económicamente por cada 100 personas de la población económicamente activa (PEA). Esta relación aumenta conforme se agrava problema de participación en las elecciones para presidente. Como se esperaba, esta diferencia promedio -estadísticamente significativa-, es más elevada en los distritos que componen el grupo de alto abstencionismo. La relación de dependencia económica alcanza a ser de 199 personas dependientes por cada 100 personas de la población económicamente activa.

\section{CONCLUSIONES}

A partir del análisis estadístico presentado en esta investigación es posible concluir que el abstencionismo electoral en los distritos de Costa Rica se distribuye desigualmente en relación a las características demográficas y económicas. En este sentido, el abstencionismo posee una correlación negativa (inversa) con respecto al índice de desarrollo social y la edad media de la población. Los distritos con menor desarrollo tienden con mayor proporción a no acudir a las votaciones. Este resultado concuerda con lo expuesto por Alfaro y Gómez (2014), en el sentido de que las regiones con menores niveles de desarrollo producen altos porcentajes de abstencionismo que se reflejan en las regiones costeras $y$ fronterizas. También Vorst (2009) concluye que el abstencionismo tiende a 
concentrarse en mayor medida en los sectores de menor nivel de escolaridad y menor nivel socioeconómico.

En aquellos distritos que apoyaron mayoritariamente al Partido Acción Ciudadana el nivel de abstencionismo distrital decreció. Se desprende de los resultados, que este partido político tuvo un efecto positivo en la disminución del abstencionismo, aspecto que se evidenció en los resultados de estas últimas elecciones presidenciales.

Los porcentajes más altos de abstención se presentan en los distritos de menor desarrollo social ubicados en las provincias de Limón y Puntarenas. Al respecto, se debe agregar que la baja densidad de población que caracteriza a estos distritos dificulta la captación de votos $y$ provoca un incremento del abstencionismo. Bonilla y Rosero (2006) plantearon que la zona atlántica parece ser un caso de estudio, pues la accesibilidad podría estar determinando un elevado abstencionismo. Es importante mencionar que en la zona urbana se logra mayor participación electoral. El abstencionismo electoral es mayor entre la población de menor edad promedio (los más jóvenes) y en donde se registra más dependencia económica. Por tanto, se puede deducir que el abstencionismo se configura como un fenómeno difícil de cuantificar con exactitud, ya que los factores que lo determinan son multivariados.

\section{REFERENCIAS}

Alfaro, R. y Gómez, S. 2014. Costa Rica: Elections Under the most Adverse context produce the Highest Political Fragmentation in 60 Years. Revista de Ciencia Política, 34(1), 125-144.

Alcubilla, E. 2000. "Abstencionismo". En: instituto Interamericano de Derechos Humanos (IIDH) (Editor). Diccionario electoral. San José.

Arias, Oscar. "Limón no será nunca más una provincia olvidada”. 27 febrero. 2008.

Bonilla y Rosero 2006. Accesibilidad a los lugares de Votación y Abstencionismo en Costa Rica. En: Capítulo 5.
Fortalecimiento de la Democracia. XII Informe Estado de la Nación en Desarrollo Humano Sostenible.

Córdoba, J. 2014. Abstencionismo no cedió a la intensidad de la Campaña. Seminario Universidad. Miércoles 5 de febrero de 2014. San José, Costa Rica.

Diccionario Electoral 2000. Instituto Interamericano de Derechos Humanos. Centro Interamericano de Asesoría y Promoción Electoral.

Fernández, M. 2014. Abstencionismo: el obstinado ganador de la elecciones en Costa. Periódico El Financiero. Sección Economía y Política. Lunes 6 de octubre de 2014, San José, Costa Rica.

González, D. 2014. Activos, pasivos y apáticos: imaginarios de la población costarricense sobre la política y la democracia. Revista Rupturas, 4(2), 100.123 .

Hernández, O. 1991. Análisis del Abstencionismo en la Elecciones Presidenciales de Costa Rica en el período 1953-1986. Anuario de Estudios Centroamericanos, 16(1-2), 117-137.

INEC (Instituto Nacional de Estadística y Censos) 2013. Encuesta Continua de Empleo: Indicadores del Mercado Laboral Costarricense, segundo trimestre 2013, San José, Costa Rica.

Lebart, L.; Morineau, A. y Piron, M. 1995. Statistique Exploratorie Multidimensionnelle, Dunod, Paris.

Lizama, C. 2012. Una Geografía Electoral del Abstencionismo en los Municipos de México (1994-2009). Universidad Nacional Autónoma de México.

MacQueen, J. 1967. Some methods for classification and analysis of multivariate observations. Proc. 5th Berkeley Symposium, 281-297.

MIDEPLAN (Ministerio de Planificación Nacional y Política Económica) 2013. Costa Rica: Índice de Desarrollo Social. Nohlen, D. 2004. La participación electoral como objeto de estudio. Rev. Elecciones. 3, 137.

Ramírez, O. 2011. Atlas electoral digital 
de Costa Rica: Documentando las elecciones de la segunda República. Reflexiones, 90(1), 3.

Raventós, C.; Fournier, M.V.; Ramírez, O. y Gutiérrez, A.L.; García, J.R. 2004. El Abstencionismo en Costa Rica, ¿quiénes son y por qué no votan los ciudadanos en las elecciones nacionales? Informe final de investigación Sociales, Universidad de Costa Rica. San José.

Vorst, C. R. (2009). Lo que fue ya no es y lo nuevo aún no toma forma: Elecciones 2006 en perspectiva histórica. América Latina Hoy, 49. 
